\title{
Informations communiquées par le Secrétariat
}

Marianne Roth | Ursula Enggist | Claudia Menolfi

\section{EXCLUSIVEMENT POUR LES MEMBRES DE L'ASP}

\section{Procès-verbal de l'assemblée}

des membres ordinaire du 23 mars 2019

Les procès-verbaux en allemand, français et italien ont été envoyés par e-mail à nos membres. Une version papier peut être commandée auprès du secrétariat ou peut être consultée dans la section réservée aux membres du site Web.

La prochaine assemblée des membres aura lieu le vendredi soir 17 avril 2020, de 17 h15 à 19 h15 à Zurich.

\section{Contrat Al}

Les psychothérapies pour enfants et mineurs, les difficultés à la naissance et pour l'insertion professionnelle peuvent, sous certaines conditions, être remboursées via l'Assurance Invalidité (AI). Sur demande, les membres de l'ASP titulaires d'une autorisation de pratique fédéral suisse ont la possibilité d’adhérer au contrat entre l'Office fédéral des assurances sociales (OFAS) et l'ASP. L'information est fournie par notre site Web ou par le bureau de l'ASP, qui reçoit également les demandes.

\section{Formulaires pour le remboursement}

Les membres peuvent commander le pratique formulaire qu'on peut remplir en ligne ou sur support papier en format PDF auprès du secrétariat, ou le télécharger dans l'espace protégé réservé aux membres du site Web, afin de facturer à leurs patients leurs honoraires.

\section{Services et tarifs}

La fiche d'information avec les directives de l'ASP sur les services et les tarifs est disponible dans la section « Fiches d'information » de notre site Web.

\section{Liste des caisses maladie}

Savez-vous quelle caisse maladie rembourse quel montant pour la psychothérapie ? Demandez la liste des caisses d'assurance maladie au secrétariat ou renseignez-vous sur notre site Web sous « Téléchargements ».

\section{Adresse électronique HIN cryptée}

Sécurisez votre adresse e-mail personnelle cryptée HIN à des conditions spéciales pour les membres de l'ASP. Ceci assure un transfert sécurisé de vos données électroniques sensibles.

\section{Offres d'emploi et locaux médicaux}

Visitez la rubrique "Actualités » sur notre site web. En plus des informations actuelles et des nouvelles de l'association, vous trouverez également des annonces pour des postes vacants et des locaux médicaux. Vous avez votre propre offre ou vous souhaitez plus d'informations? Veuillez contacter le secrétariat pour obtenir de plus amples renseignements sur l'emplacement sur notre site Web.

\section{Entrée de nos membres dans PsyReg}

Tous nos membres à part entière sont inscrits au PsyReg à titre de psychothérapeutes $(\mathrm{h} / \mathrm{f})$ reconnu(e)s au niveau fédéral. Les membres nouvellement admis sont automatiquement signalés à l'Office fédéral de la santé publique (OFSP) pour leur inscription au registre. Si vous souhaitez modifier votre entrée, vous devez le solliciter directement au OFSP. Les directions cantonales de la santé sont responsables de la validité de l'autorisation d'exercer cantonales dans le PsyReg.

\section{Devoir de formation continue des membres}

Les psychothérapeutes $(\mathrm{h} / \mathrm{f})$ sont légalement tenus de suivre des cours de formation continue sur une base continue. Cela sert à la formation scientifique postgrade, à létude de la théorie ainsi quà l'extension et à l'approfondissement des compétences pratiques. Vous trouverez de plus amples informations et notre vaste programme de formation continue sur notre site Web sous la rubrique « Formation postgrade \& Formation continue».

\section{Informations relatives à la psychothérapie}

Dans la section réservée aux membres de notre site Web, vous trouverez les publications qui sont contraignantes (de lecture obligatoire) en tant que membre de l'ASP. En plus des règlements, des fiches d'information font référence à diverses questions sur des domaines spécifiques de la profession de psychothérapeute. Utilisez notre secrétariat comme centre d'information.

\section{Archivez votre documentation}

Vous envisagez de vous retirer de la vie professionnelle active et souhaitez archiver vos dossiers en toute sécurité ? Souhaitez-vous que vos documents rassemblés soient conservés en toute sécurité dans le sens d'un règlement successoral ? L'ASP peut exclusivement offrir à ses membres une solution économique et sûre. De plus amples informations peuvent être obtenues auprès de notre secrétariat.

\section{ASSURANCES}

\section{Forfait tout compris}

Grâce au contrat de groupe avec notre agence dassurance Swiss Quality Broker AG, nous sommes en mesure d'offrir à nos membres un forfait exclusif de services d'assurance à bas prix comprenant les offres suivantes $\cdot$ Assurance maladie $\cdot$ Assurance de responsabilité civile professionnelle $\cdot$ Assurance de protection juridique - Assurance des choses de lentreprise - Assurance-maladie et accidents - Assurance perte de gain Les membres de l'ASP peuvent également bénéficier de primes avantageuses pour leur assurance voiture/moto, assurance ménage, assurance responsabilité civile privée et lassurance bâtiment auprès de ZURICH. Vous trouverez de plus amples informations dans l'espace réservé aux membres sur notre site Web. 
Recommandation supplémentaire : C Caisse de pension (deuxième pilier)

La Fondation Pro Medico Zurich offre aux membres de l'ASP la possibilité d'une prévoyance professionnelle flexible en tant que prévoyance collective du deuxième pilier. Il s'agit d’offres financièrement intéressantes pour la prévoyance vieillesse individuelle des travailleurs indépendants et de leur personnel.

\section{INFORMATIONS GÉNÉRALES}

Notre site Web www.psychotherapie.ch contient toutes les informations pertinentes pour nos membres dans une zone protégée avec accès par mot de passe. Les dates de la prochaine assemblée des membres, les colloques pour la conférence de la Charte, les solutions d'assurance, etc. y sont également disponibles. Nous vous recommandons une visite régulière.

Outre des informations sur notre organisation et sur la psychothérapie en général, notre site Web contient également des informations destinées au grand public sur les sujets suivants : - Politique professionnelle et développement de carrière · Formation postgrade \& continue $\cdot$ Science et recherche Des appels d’offres pour des manifestations et événements d'actualité, des cours de formation continue, des conférences, etc. sont disponibles sur notre site Web sous la rubrique « Agenda ».

\section{Actualités}

Découvrez par exemple l'état d'avancement du modèle de la prescription et d'autres sujets d'actualité sur notre site Web sous la rubrique «Actualités ».

Vous avez un souhait ou une suggestion ? N’hésitez pas à communiquer avec notre bureau.

\section{PUBLICATIONS}

Le magazine à jour - Psychotherapie-Berufsentwicklung est lorgane d'information bilingue (allemand et français) de l'ASP avec un contenu de politique professionnel et spécialisé. Il se considère comme un lien entre nous en tant qu'association professionnelle et nos membres ainsi que tous les lecteurs intéressés par la psychothérapie.

La revue Psychotherapie-Wissenschaft est dédiée au développement de la psychothérapie. Les contributions à la pratique et à la recherche favorisent les échanges interdisciplinaires sur des questions fondamentales telles que l'indication, la méthodologie et l'efficacité.

Les deux éditions sont publiées deux fois par an. D’ailleurs, les articles des deux revues peuvent être consultés sur Internet en version numérique à l'adresse suivante : www.psychotherapie-wissenschaft.info

\section{Annonces}

Profitez de l'occasion pour publier vos notifications ou annonces dans nos revues. Les articles publiés s'adressent aux psychothérapeutes (h/f) praticiens ainsi qu'aux experts de la science, de la recherche et de la pratique. Renseignez-vous auprès du bureau sur les options de publication, les prix et les méthodes de publication.

\section{Bulletin d'information}

Notre bulletin d'information vous tient au courant des activités actuelles de l'association et de l'évolution de la politique professionnelle. Il est publié quatre fois par an.

\section{QUESTIONS D'ORGANISATION}

\section{Médias}

Le bureau est heureux de recevoir les demandes de renseignements des représentants des médias. Notre association regroupe des praticiens expérimentés sur différents sujets et méthodes psychothérapeutiques, y compris les sujets actuels et méthodes thérapeutiques actuelles.

\section{Membres du comité de l'ASP}

- Gabriela Rüttimann, Présidente

- Veronica Defièbre, vice-présidente, responsable de la Conférence de la Charte

- Sandra Feroleto, déléguée pour la Suisse romande

- Nicola Gianinazzi, délégué pour la Suisse italienne

- Peter Schulthess, rédacteur en chef à jour et psychothérapeute-scientifique

\section{Bureau}

- Marianne Roth, directrice générale

- Ursula Enggist, Secrétariat

- Claudia Menolfi, Secrétariat

\section{Contact :}

Téléphone : 0432689300

Courriel : marianne.roth@psychotherapie.ch

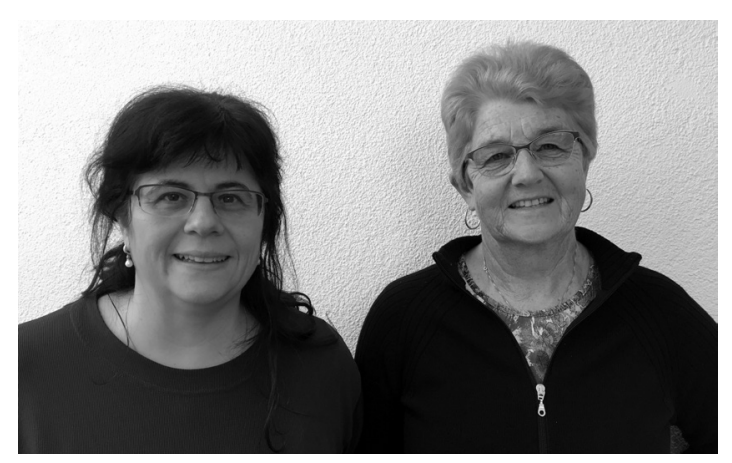

(de gauche à droite :) Claudia Menolfi et Ursula Enggist 